\title{
Characterization of Turbiditic Oil Reservoirs Based on Geophysical Models of their Formation
}

Semi-Annual Technical Progress Report

Reporting Period Start Date: January 1, 2003

Reporting Period End Date: March 31, 2003

Authors: Roger T. Bonnecaze and Srivatsan Lakshminarasimhan

June 2003

DOE Award \#: DE-FC26-01BC15352

Department of Chemical Engineering

The University of Texas at Austin 


\section{Disclaimer}

This report was prepared as an account of work sponsored by an agency of the United States Government. Neither the United States Government nor any agency thereof, nor any of their employees, makes any warranty, express or implied, or assumes any legal liability or responsibility for the accuracy, completeness, or usefulness of any information, apparatus, product, or process disclosed, or represents that its use would not infringe privately owned rights. Reference herein to any specific commercial product, process, or service by trade name, trademark, manufacturer, or otherwise does not necessarily constitute or imply its endorsement, recommendation, or favoring by the United States Government or any agency thereof. The views and opinions of authors expressed herein do not necessarily state or reflect those of the United States Government or any agency thereof. 


\section{Contents}

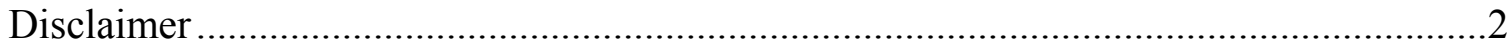

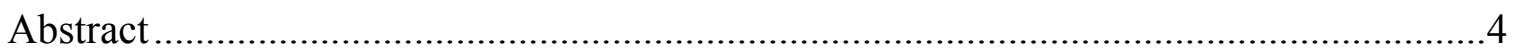

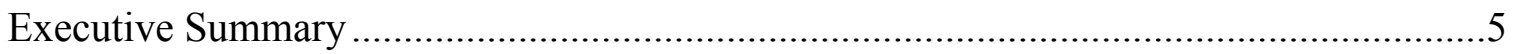

Methodology - Numerical Simulation of Turbidity Current ..........................................6

Results and Discussion - Numerical Simulation of Turbidity Current ...........................10

Methodology - Preliminary Sensitivity Study of Parameter Estimation

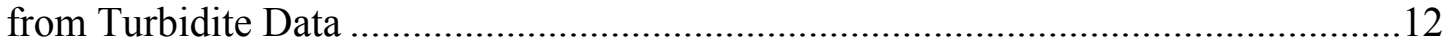

Results and Discussion - Preliminary Sensitivity Study of Parameter Estimation from Turbidite Data .......................................................................................... 16

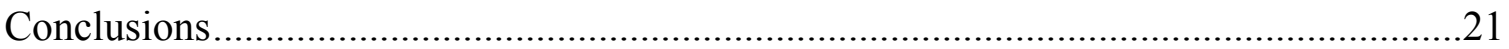

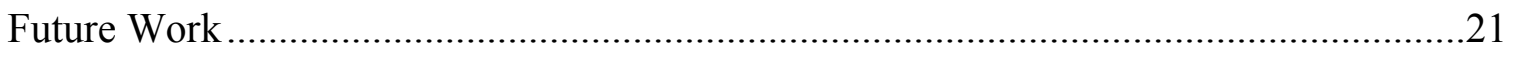

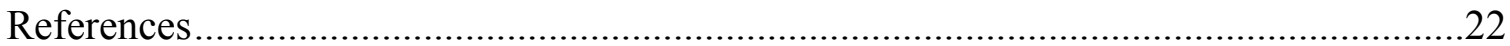




\begin{abstract}
Two aspects of the characterization of turbiditic oil reservoirs based on geophysical models of their formation are discussed in this report. First, we have developed a new, more accurate and computationally faster finite-element method (FEM) for simulating the flow and deposition of turbidity currents. Although a finite volume method had been presented and discussed in a previous report, it was discovered to be insufficient for our purposes of simulating turbidity flows. The new method allows variable grids near the regions of large deposition, which are of most interest, and numerically results in banded, sparse matrices that are much faster to solve. Examples of the success of the method are presented.

In the second part of this report, we present and discuss a preliminary study on the feasibility of matching the results of a sediment transport model to field data. With the simulation of the turbidity current we can create an entire turbiditic deposit. This requires the initial conditions of the flow, such as the amount of sediment, the volume or flow rate of the current, etc, which are of course unavailable. This requires an estimate of the initial conditions of the flow, which can be determined from limited data from the deposit. We used the Excel optimization routine Solver to reproduce a one-dimensional algebraically simulated deposit with and without measurement noise. Results indicate that such matching is feasible, provided that the noise is below certain thresholds, dependent on the size of the deposit and the number data points constraining the parameter estimation.
\end{abstract}




\section{EXECUTIVE SUMMARY}

Our method for characterizing turbiditic reservoirs based on geophysical models of their formation relies on two parts. First, we need a model for the formation of the deposit, which for these complex flows takes the form of a computational fluid mechanics simulation of the dynamics and deposition of turbidity flows. With the simulation and the initial conditions of the flow (sediment loading, volume of current, etc.), and entire deposit can be constructed. However, these initial conditions are never known but must be inferred from field data. Thus, the second aspect of this work is the estimation of these initial conditions from a few measurements of the deposit. In this report, we discuss both these aspects.

In previous reports we had presented and discussed the simulation of turbidity currents using a finite-volume method (FVM). Over the last several months, it has become apparent that this method was not viable for the accurate and rapid simulation of turbidity flows. The deposition occurs over a small region, which needs very fine grids to resolve. Variable grids with the FVM have proved to unsuccessful, and we thus had to revise our codes to implement a much better finite-element method (FEM) with our SIMPLER algorithms discussed in previous reports. This FEM approach is significantly more numerically stable, it allows the variable grid necessary for good resolution of the deposition and it is computationally faster. Preliminary results with this new method are presented.

We also completed a preliminary study of the estimation of the initial conditions of a turbidity flow from the deposit (here generated synthetically from an algebraic model). Such problems are well known in geophysics, usually as inverse problems. Parameter estimation is often difficult in many geophysical systems due to the illconditioned nature of the problem and measurement noise. Here we explore these sensitivities by studying the parameter estimation using a simple forward model of the deposition. Optimization was performed to examine whether an inverse method is feasible to predict the sediment density of an entire turbidite deposit based on a few point observations. We have demonstrated that an inverse method is feasible by performing optimizations using Excel Solver. A FORTRAN version of the Solver algorithm is available for ultimate implementation with the computational simulations. We must use the scaling option in the solver for convergence, and we speculate such a procedure will be necessary in the final application. When adding both additive and multiplicative perturbations to the density, the average relative error in the returned values will increase with the size of the perturbation. To perform the optimization we must choose fairly accurate starting values when the number of measurement points is small. The insight and techniques we find successful here will be implemented with the more complex FEM simulator of the deposition process. 


\section{METHODOLOGY - NUMERICAL SIMULATION OF TURBIDITY CURRENT}

\section{Introduction}

We have previously been simulating the two-dimensional flow of turbidity currents using a finite-volume method. In our preliminary computations, we discovered that the accurate calculation of the dimensions of deposition within reasonable computation time requires the use of variable grid, i.e. finer grid elements along the bottom and close to inlet and coarser elements in other regions. However, the finitevolume method has turned out to be unsuitable for such mesh refinement. There is in fact a net loss of resolution by using a variable grid due to the loss of second order spatial accuracy in the method. Thus, we had to abandon the finite-volume method for a much more accurate finite-element method (FEM). A significant amount of time has been spent rewriting the simulation for this conversion.

The FEM offers the following advantages: (i) as FEM solves the weak variational form of the differential equation, it is generally more stable than FVM. This allows simulations of turbidity currents and there deposits for longer times than previously; (ii) the highest degree of derivatives in the weak variational form of the differential equations is one less order than in the original equations. This contributes to the improvement in accuracy of the solution, particularly in the case of particle phase velocity calculations; (iii) the finite-element based solution procedure to solve particle phase mass balance equation shows marked improvement in particle concentration profile when compared to that obtained from FVM based method; and lastly, (iv) the FEM yields symmetric banded matrices which require less computation time to solve than unsymmetric banded matrices obtained from FVM. For these reasons, we decided to employ FEM to solve the model equations.

The model equations consisting of six differential equations in as many unknown variables are solved using FEM in three stages. In the first stage, the suspension phase mass and momentum balance equations are solved for suspension velocity and pressure using SIMPLER-like algorithm. Next, the particle phase momentum balance equations are solved for particle velocity using the suspension velocity calculated in the first stage. In the final stage, the particle phase mass balance equation is solved for particle concentration using particle velocity.

The organization of this report is as follows. The mathematical model that governs the flow is presented in the next section. Although this has presented earlier, we do so in here in a slightly different form for the FEM solver. The algorithm employed in each of the three stages is described below and results and a discussion follow.

\section{Suspension balance model}

The flow of a suspension consisting of mono-dispersed particles released from a constant flux per width source of strength $q$ is considered. The height of the source is $h_{\text {in }}$. A parabolic profile whose flow rate is $q$ is prescribed for suspension velocity at the inlet. The volume fraction of particles in the suspension is $\varphi_{\text {in. }}$. The densities of particles and suspending fluid are $\rho_{\mathrm{p}}$ and $\rho_{\mathrm{a}}$, respectively. The suspension is released on a horizontal planar surface into an ambient fluid whose density is same as that of the suspending fluid $\rho_{\mathrm{a}}$. 
The flow of turbidity currents is modeled as a two-phase flow, consisting of suspension phase and particle phase (Nott and Brady 1994). The suspension phase is characterized by velocity $\langle\boldsymbol{u}\rangle$ and pressure $p_{f}$. The particle phase is characterized by the velocity vector $\langle\boldsymbol{u}\rangle_{p}$ and the volume fraction of particles $\varphi$. The principles of conservation of mass and momentum are applied to each phase to obtain differential equations for the velocity of each phase and particle concentration. In addition, Nott and Brady (1994) also apply the principle of conservation of energy to the entire current to obtain an equation for suspension temperature $T$, which is a measure of the fluctuations in suspension velocity. The inclusion of suspension temperature into the model plays a crucial role in problems where the suspension strain rate $\gamma=0$ at any point within the domain of flow (Morris \& Brady 1998; Morris \& Boulay 1999). However, in the case of deep ocean turbidity currents, the strain rate does not vanish at any point within the flow. Hence, the suspension energy balance equation is dropped from the model developed to study the flow of turbidity currents. Furthermore, inertial effects are not taken into account in this study. This not only enables the understanding of the physics of viscous turbidity currents, but also applies to certain special types of turbidity flows called debris flow.

The length and velocity scales used to non-dimensionalize the corresponding variables are given by $L_{0}=q / v_{s}$ and $U_{0}=v_{s} f\left(\varphi_{i n}\right)$, respectively. Here, $v_{s}$ is the Stokes settling velocity and $f$ represents hindered settling function. The length scale $L_{0}$ represents the characteristic distance traveled by a particle. The suspension pressure is scaled by $P_{0}=\eta U_{0} / L_{0}$ where $\eta$ is the viscosity of suspending fluid. The particle concentration is scaled by inlet concentration $\varphi_{i n}$.

There are two dimensionless ratios involved in the mathematical description of the problem. They are (i) the buoyancy number $N_{B}$ which is defined as the ratio of buoyancy to viscous forces, given by

$$
N_{B}=\frac{\left(\rho_{p}-\rho_{a}\right) g \phi_{i n}}{\eta} \frac{L_{0}^{2}}{U_{0}}
$$

and (ii) the volume fraction of particles at the inlet $\varphi_{i n}$.

The scaled equations governing the dynamics of suspension phase are given by

$$
\begin{gathered}
\nabla \cdot\langle\boldsymbol{u}\rangle=0 \\
-\nabla p_{f}+\nabla^{2}\langle\boldsymbol{u}\rangle+\boldsymbol{M}(\langle\boldsymbol{u}\rangle)=0
\end{gathered}
$$

where the vector $\boldsymbol{M}$ is given by

$$
\boldsymbol{M}=\left(\begin{array}{c}
-\frac{\partial \pi}{\partial x}+2 \frac{\partial}{\partial x}\left(\eta_{p} \frac{\partial\langle\mathrm{u}\rangle}{\partial x}\right)+\frac{\partial}{\partial y}\left(\eta_{p} \frac{\partial\langle\mathrm{u}\rangle}{\partial y}\right)+\frac{\partial}{\partial y}\left(\eta_{p} \frac{\partial\langle\mathrm{v}\rangle}{\partial x}\right) \\
-N_{B} \phi-\lambda_{2} \frac{\partial \pi}{\partial y}+\frac{\partial}{\partial x}\left(\eta_{p} \frac{\partial\langle\mathrm{u}\rangle}{\partial y}\right)+\frac{\partial}{\partial x}\left(\eta_{p} \frac{\partial\langle\mathrm{v}\rangle}{\partial x}\right)+2 \frac{\partial}{\partial y}\left(\eta_{p} \frac{\partial\langle\mathrm{v}\rangle}{\partial y}\right)
\end{array}\right)
$$

In these equations, the angular brackets $<$ represent averaging of the variables over length scale which is much greater than molecular dimension, but much less than 
any length scale of the problem. $\lambda_{2}=0.8$ is a constant which represents the ratio of normal stress difference in gradient to flow directions. In addition, $\pi(\varphi)=\eta_{n}(\varphi) \gamma$ represents particle pressure, $\gamma$ represents strain rate, $\eta_{p}$ and $\eta_{n}$ the particle phase and normal stress viscosity coefficients. The $\varphi$-dependent material functions $\eta_{p}, \eta_{n}$ and $f$ are obtained from Morris \& Brady (1998); Morris \& Boulay (1999); Davis \& Acrivos (1985) respectively, and are given by

$$
\begin{gathered}
\eta_{p}=(1-x)^{-2}-1, \\
\eta_{n}=0.8 x^{2}(1-x)^{-2}, \\
f=(1-x)^{-4}, \quad \text { where } \\
x=\frac{\phi_{\text {in }} \phi}{\phi_{m}} .
\end{gathered}
$$

Here, $\varphi_{m}=0.63$ corresponds to volume fraction of mono-dispersed particles at maximum packing.

The equations governing the motion of particle phase are given by

$$
\begin{gathered}
\frac{\partial \phi}{\partial t}+\nabla \cdot\left(\phi\langle\boldsymbol{u}\rangle_{p}\right)=0 \\
\langle\boldsymbol{u}\rangle_{p}=\langle\boldsymbol{u}\rangle+g(\phi) \boldsymbol{M}(\langle\boldsymbol{u}\rangle) \\
g(\phi)=\frac{N_{B}}{f\left(\phi_{i n}\right)} \frac{f(\phi)}{\phi}
\end{gathered}
$$

Boundary conditions applied to this system of equations are as follows. (i) no slip condition for suspension and particle velocities along bottom surface, (ii) prescribed velocities for both velocities along inlet, (iii) traction-free boundary conditions along far boundaries, i.e.

$$
\begin{aligned}
-p_{f}+2 \frac{\partial\langle\boldsymbol{u}\rangle}{\partial x}=0, & \frac{\partial\langle\boldsymbol{v}\rangle}{\partial x}=0, \quad \text { along far right boundary } \\
-p_{f}+2 \frac{\partial\langle\boldsymbol{v}\rangle}{\partial y}=0, & \frac{\partial\langle\boldsymbol{u}\rangle}{\partial y}=0, \quad \text { along top boundary. }
\end{aligned}
$$

Initial condition for $\varphi$ is given by $\varphi=0$ everywhere except along the inlet where $\varphi=1$.

\section{Numerical algorithm}

The model equations are solved in three stages sequentially. The suspension phase mass and momentum conservation equations are solved in the first stage using transient SIMPLER. The particle phase momentum conservation equations are solved in the second stage. Finally, the particle mass conservation equation is solved in stage 3 . The algorithms developed for each stage is presented below.

\section{Stage 1: Suspension phase equations}

The SIMPLER (Semi-Implicit Method for Pressure Linked Equations: Revised) algorithm is used to solve the steady momentum (3) and mass (2) conservation equations. This algorithm is based on uncoupling pressure from velocity. The pressure variable 
adjusts itself so as to enable the velocity field to satisfy continuity equation (2). It has no impact on the vorticity component of the velocity field. This aspect is exploited by SIMPLE algorithm to calculate velocity and pressure. The steps involved in this iterative algorithm as given below:

1. Start with an initial guess for suspension velocity field.

2. Set pressure $p_{f}=0$ and solve suspension momentum balance equation (3) for $\langle\hat{\boldsymbol{u}}\rangle$, which has the same vorticity as $\langle\boldsymbol{u}\rangle$.

3. Obtain an equation for pressure using continuity constraint and $\langle\hat{\boldsymbol{u}}\rangle$. Solve for pressure $p_{f}^{*}$.

4. Using pressure $p_{f}^{*}$, solve momentum equation (3) to obtain $\left\langle\boldsymbol{u}^{*}\right\rangle$.

5. Obtain an equation for pressure correction $\delta p_{f}$ using continuity constraint and velocity $\left\langle\boldsymbol{u}^{*}\right\rangle$.

6. Solve momentum equations using pressure correction to obtain velocity correction.

7. Update velocity field.

8. Repeat steps (2)--(7) until convergence.

The crucial steps of this algorithm are (3) and (5) which pertain to the derivation of an equation for pressure using continuity equation.

An equation for pressure can be derived in two ways. (i) The discretized continuity and momentum equations can be manipulated to obtain a discretized equation for pressure. In this case, the constraints stemming from the discretized continuity are applied directly on the velocity field. In order to avoid overconstraining the velocity field, the functions weighting the continuity equation, namely pressure interpolating polynomials, are chosen to be one degree less than the polynomials interpolating velocity components (Williams \& Baker, 1966). The use of lower degree polynomial is advantageous in that it saves computation time. However, this method fails for problems with flow through boundaries. (ii) Alternately, the continuous momentum and continuity equations can be manipulated to obtain a continuous Poisson type equation for pressure. This approach requires the use of equal order interpolating polynomials for velocity and pressure. This method of deriving pressure equation is able to handle problems with flow through boundaries, which we have adopted.

The velocity obtained by solving the steady momentum and continuity equations using SIMPLER algorithm did not satisfy mass conservation equation. The reason for this is due to the error involved in the application of boundary conditions for pressure. Nonino \& Comini (1997) developed an algorithm to solve transient Stokes equations which got rid of the error associated with pressure boundary conditions. Based on the algorithm for transient Stokes equations, Codina \& Blasco (1997) developed a fractionalstep algorithm to solve steady Stokes equations. Upon implementing both of these algorithms, I found the transient algorithm better for two reasons -- (i) marginally better accuracy in pressure profile, and (ii) less computation time. Hence, we decided to obtain steady-state velocity and pressure by solving unsteady momentum and mass conservations, i.e. 


$$
\begin{gathered}
\nabla \cdot\langle\boldsymbol{u}\rangle=0, \\
\frac{\partial\langle\boldsymbol{u}\rangle}{\partial t}=-\nabla p_{f}+\nabla^{2}\langle\boldsymbol{u}\rangle+\boldsymbol{M}(\langle\boldsymbol{u}\rangle) .
\end{gathered}
$$

\section{Stage 2: Particle phase momentum equations}

In the viscous regime, the particle phase momentum equations are not coupled. Hence, the solution procedure using FEM to obtain particle phase velocity is straightforward. The derivation of the weak variational form of the differential equations directly leads to FEM-based calculation of particle velocity. This procedure yields more accurate solution for particle velocity than that obtained from the finite-volume-based method.

\section{Stage 3: Particle phase mass conservation equation}

The application of mass conservation for particle phase results in a time dependent hyperbolic equation. In order to improve the accuracy of the particle concentration profile, we decided to develop a code to solve this equation using FEM. Based on the FEM-based flux-corrected transport algorithm given by Georghiou et al $(1999,2000)$, we developed an algorithm to solve the particle mass equation (9).

\section{RESULTS AND DISCUSSION - NUMERICAL SIMULATION OF TURBIDITY CURRENT}

This section presents results obtained from the simulation of turbidity currents using the recently developed FEM-based algorithm. The $x$ - and y- axes represent the direction of motion and vertical direction respectively. As channelized flows are considered here, the simulations are confined to two dimensions (i.e., $x y$ plane). The current is injected at $x=0$ at all times and the height of the current at the inlet is $h_{\text {in }}=$ 0.4 . The particles in the current are uniformly suspended at the inlet.

Figure 1 shows the grid distribution, suspension phase velocity, suspension pressure and particle concentration for $N_{B}=0$ and $\varphi_{\text {in }}=0.1$ at dimensionless time $t=2$. Variable grid is chosen so as to obtain greater resolution in regions close to inlet and bottom surface. The use of variable grid enables simulation over large domains using a fewer number of elements. The suspension velocity shows predominantly radial expansion, as would be expected of flow with neutrally buoyant particles. The suspension pressure shows a predominantly radial variation except in regions close to bottom surface and vertical line along inlet. This deviation from radial profile in these regions is due to zero velocity condition enforced on suspension velocity. The particle concentration profile also shows a near radial symmetry owing to neutrally buoyant particles. Another interesting observation is that at any fixed radial distance from the inlet, particle concentration is lower in the central regions close to 45-degree line where suspension velocity is largest.

Figure 2 shows the grid distribution and particle concentration for $N_{B}=100$ and

$\varphi_{\text {in }}=0.1$ at increasing times. Particles clearly collect at the bottom of the surface, beginning the formation of the deposit. 
(a) Grid Distribution

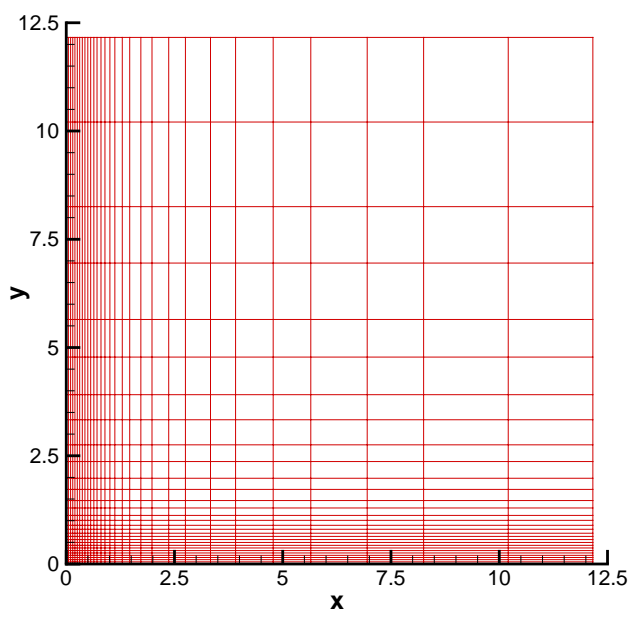

(c) Suspension pressure

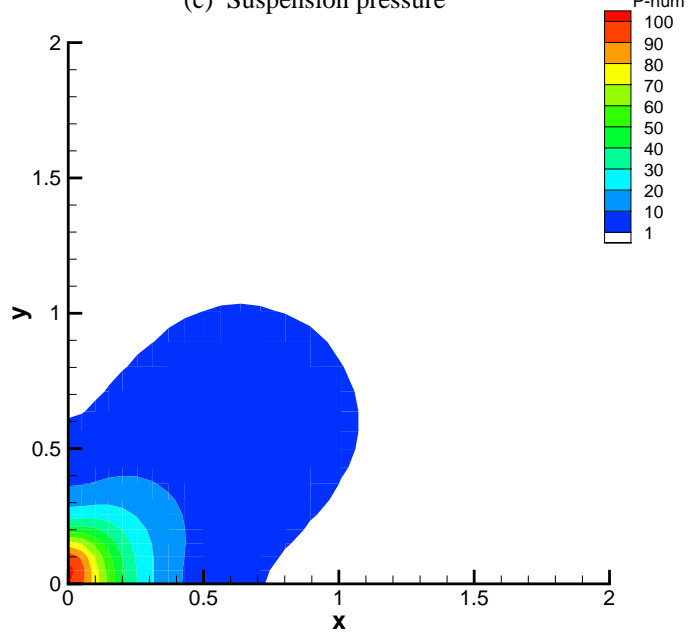

(b) Suspension phase velocity

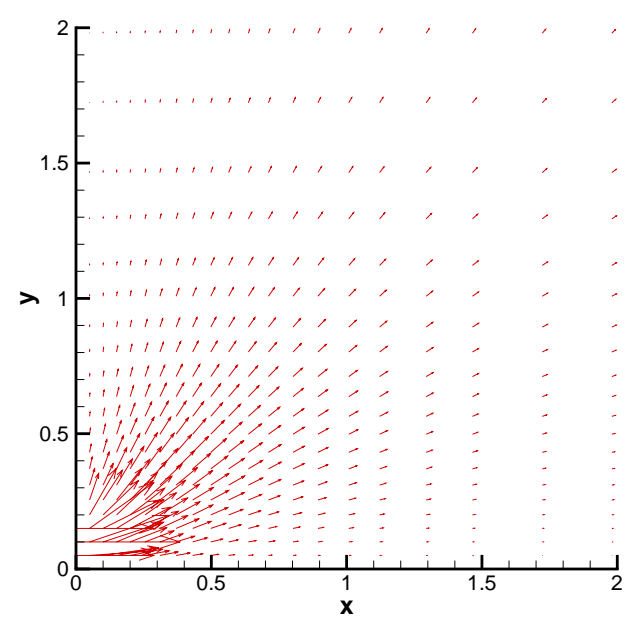

(d) Particle concentration

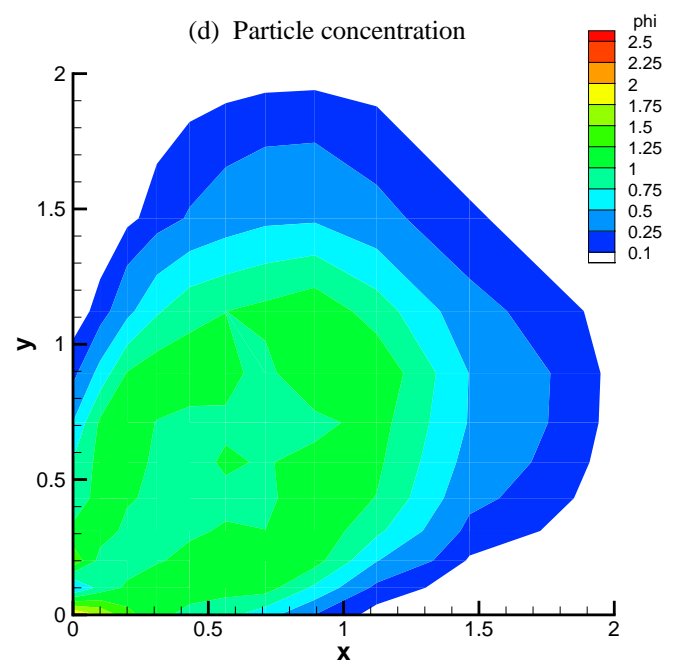

Figure 1: Results from simulation of turbidity flow with $N_{B}=0$ and $\varphi_{i n}=0.1$ at $t=2$. (a) Grid distribution, (b) suspension velocity, (c) suspension pressure and (d) particle concentration. Note that the length of the arrows in (b) indicate relative speed of flow. In (d), $\varphi=6.3$ corresponds to a maximally random packed suspension. 
(a) Grid Distribution

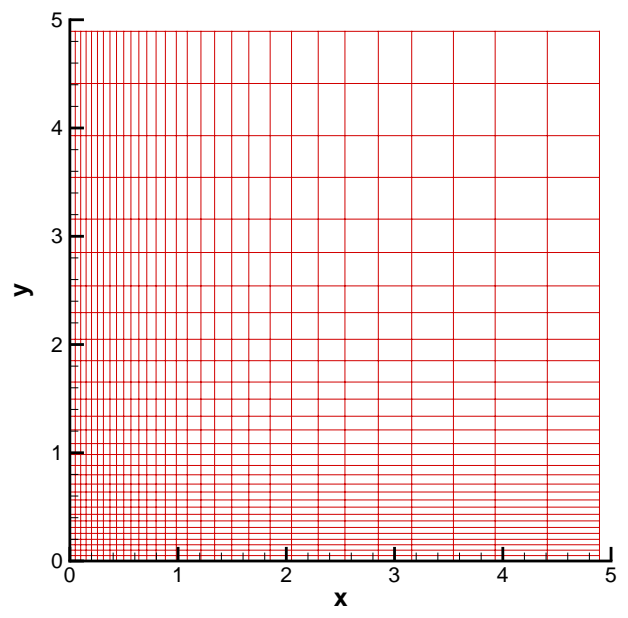

(c) Particle concentration at $\mathrm{t}=1$

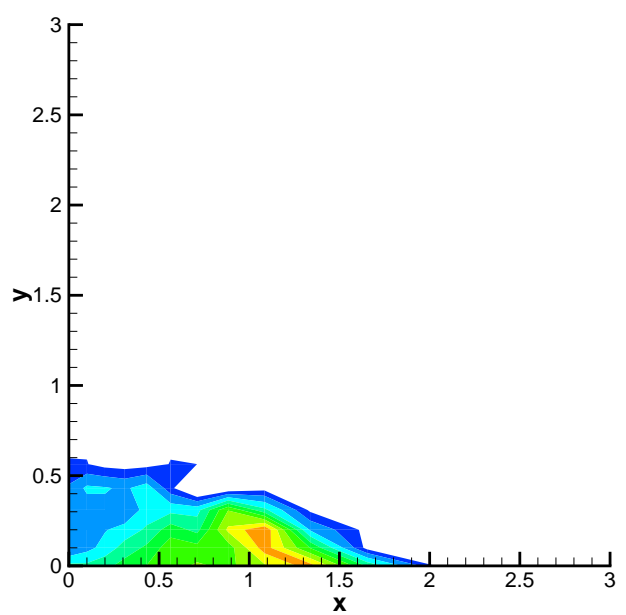

(b) Particle concentration at $\mathrm{t}=0.5$

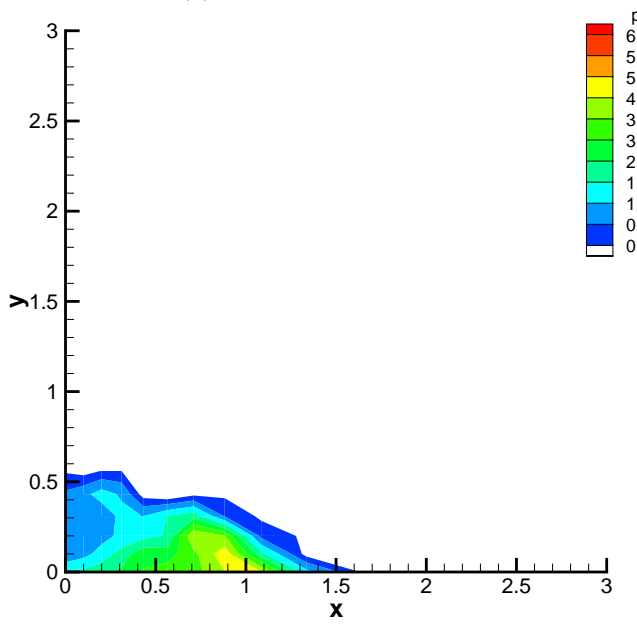

(d) Particle concentration at $\mathrm{t}=2$

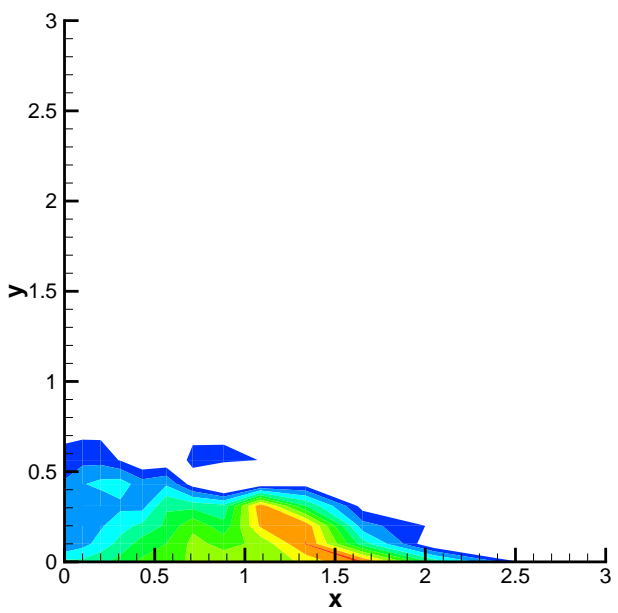

Figure 2: Results from simulation of turbidity flow with $N_{B}=100$ and $\varphi_{\text {in }}=0.1$ including (a) the grid distribution at times (b) $t=0.5$, (c) ) $t=1.0$ and (d) $t=2.0$. Note that $\varphi=6.3$ correspond to a maximally random packed suspension.

\section{METHODOLOGY - PRELIMINARY SENSITIVITY STUDY OF PARAMETER ESTIMATION FROM TURBIDITE DATA}

\section{Introduction}

With the simulation of the turbidity current we can create a turbiditic deposition. This requires the initial conditions of the flow, such as the amount of sediment, the volume or flow rate of the current, etc, which are of course unavailable. This information, however, can be inferred from a small number of samples or cores taken from a turbidite by solving a parameter estimation problem. Such problems are well known in the geophysics, usually as inverse problems. Parameter estimation is often difficult in many geophysical systems due to the ill-conditioned nature of the problem and measurement noise. Here we explore these sensitivities by studying the parameter estimation using a simple forward model of the deposition. Optimization was performed 
to examine whether an inverse method is feasible to predict the sediment density of an entire turbidite deposit based on a few point observations. The insight and techniques we find successful here will be implemented with the more complex FEM simulator of the deposition process.

We constructed an algebraic target function for the spatial distribution of a turbidite deposit developed by Bonnecaze et al. (1996), which is shown below. The target function is deposit density versus distance from the sediment source. The target function requires two parameters, $q$ and $\phi$ the initial volume per unit width of the current and initial volume fraction of particles, respectively. Here the deposit is assumed onedimensional (from a two-dimensional turbidity flow) with grains of uniform size. Next, we select a few point measurements at different locations from the target, and define a function $\theta$, expressed as

$$
\theta=\sum(\text { predicted density of deposit }(\phi, q)-\text { target density of deposit })^{2} .
$$

The sum in this equation is over the total number of selected points and the predicted density is from the same model as was used to generate the target. We search the $(\phi, q)$ parameter space $\hat{q}$ and $\hat{\phi}$ that minimize $\theta$. The returned $\hat{q}$ and $\hat{\phi}$ at the minimum should perfectly match the $q$ and $\phi$ for perfect data, and closely match them for imperfect data due to measurement noise. With estimates for these parameters, one could then construct the entire turbidite deposit, honoring the core the data. This is a non-linear optimization problem for which we use Microsoft Excel Solver to perform the minimization. A FORTRAN version of this solver is available for implementation with the more complex FEM simulator.

\section{Description of the deposition model}

The density of a turbidite deposit, defined as the mass per unit area, is a measure of the thickness of the deposit. It would be valuable to be able to estimate this density and the particle-size distribution throughout a deposit from only a few point measurements. From this we might be able to determine the density over the entire reservoir, which would be useful in subsequent development of the field. We would also infer the initial condition of the current that produced the deposit, that is, the volume fraction distributions and the initial volume of the current.

Based on an analysis of the transport equations for a turbidite formed from a finite volume release in two-dimensions and comparisons to exact numerical simulations, Bonnecaze et al., (1996) gave an approximate, but convenient, algebraic representation of the density of the deposit composed of one size particle or many different sized particles. Here we consider only the case for a well-sorted or single particle size deposit. For a gravity current composed of uni-diameter particles the density of the deposit $w$ is given by,

$$
w=\rho_{p} q^{1 / 2} \phi \beta_{q}^{2 / 5} W\left(x_{D}\right)
$$

where $x_{D}$ is the dimensionless distance from the source of the current given by 


$$
x_{D}=x /\left(\beta_{q}^{-2 / 5} q^{1 / 2}\right)
$$

and

$$
\beta_{q}=v /\left(g_{0}^{\prime 1 / 2} q^{1 / 4}\right)
$$

where $x$, is the dimensional downstream distance, $v$ is the settling velocity of the particle and $q$ is here the initial volume per unit width of the current. The reduced density $g_{0}^{\prime}$ is a function of the volume fraction of particles $\phi$ is defined as

$$
g_{0}^{\prime}(\phi)=\left[\rho_{c}(\phi)-\rho_{a}\right] g / \rho_{a}
$$

where $\rho_{c}, \rho_{a}, \rho_{p}$ are the densities of a well-mixed suspension, the ambient fluid and the particle respectively, where

The function $W$ is a "master" function that it fit to a numerical solution. $W$ is given by

$$
W\left(x_{D}\right)=0.802 /\left(1+0.683 x_{D}^{2}+0.017 x_{D}^{8}\right) .
$$

\section{Parameter estimation}

Parameters other than $q$ and $\phi$ are listed in Table 1. With these values, Eq. (17) becomes

$$
w(x)=1.89 \times 10^{2} q^{2 / 5} \phi^{4 / 5} /\left(1+5.1668 \times 10^{-3} q^{-5 / 6} \phi^{-2 / 5} x^{2}+5.5637 \times 10^{-11} q^{-24 / 5} \phi^{-8 / 5} x^{8}\right)
$$

Table 1. Parameters used for calculation

\begin{tabular}{|l|l|}
\hline Initial volume $q$ & $10^{4} \mathrm{~m}^{2}$ \\
\hline Initial volume fraction of particle $\phi$ & 0.02 \\
\hline Density of particle $\rho_{p}$ & $2.65 \times 10^{3} \mathrm{~kg} / \mathrm{m}^{3}$ \\
\hline Diameter of particle $d$ & $10^{-4} \mathrm{~m}$ \\
\hline Gravity $g$ & $9.81 \mathrm{~m} / \mathrm{s}^{2}$ \\
\hline Density of ambient fluid (water) $\rho_{a}$ & $1.0 \times 10^{3} \mathrm{~kg} / \mathrm{m}^{3}$ \\
\hline Viscosity of ambient fluid (water) $\mu$ & $1.0019 \times 10^{-3} \mathrm{~Pa} \cdot \mathrm{s}$ \\
\hline
\end{tabular}

We take the preset conditions to be $q=10^{4} \mathrm{~m}^{2}$ and $\phi=0.02 . w(x)$ with these values is the target deposit. We use Eq. (22) to compute the density of this target deposit $w(x)$ along the $x$ direction. Figure 3 shows the target deposit. For the optimization step, we load all the variables and measured density $w(x)$ in Excel cells (the target function had 
100 points) and let Excel calculate model output as predicted values. The objective function is given by

$$
\theta=\min _{\phi, q}\left[\sum_{i=1}^{M}\left(\hat{w}\left(x_{i}, \phi, q\right)-w\left(x_{i}\right)\right)^{2}\right],
$$

where $x_{i}$ is the preset location. $M$ is the number of measurement points, which are taken to be uniformly spaced between the source and the furthermost tip of the deposit in all cases. In the first experiment $M=60 . q_{0}$ and $\phi_{0}$ are the starting values for the Solver optimization.

Solver gives satisfying results, which means that $\theta$ is small and the returned values of $\hat{q}$ and $\hat{\phi}$ are almost the same as we used for the target case. Thus, the predicted density agrees well with that of the target. We did find that Eq. (22) is poorly scaled, because the values of $q$ and $\phi$ are substantially different from each other. This means that the value of the objective function changes dramatically when one of both of the two decision variables $q$ and $\phi$ change a little. See the objective function in Figure 4. The range of the vertical axis is very large. For this kind of model we had to use the Automatic Scaling box in the Solver Options dialog box, which causes Solver to automatically rescale both the variables and function values internally before solution. Using the scaling option, Solver returned the target $q$ and $\phi$ that were almost exactly equal to the preset values. This preliminary study alerts us that a similar rescaling probably will be necessary when using the FEM simulator for the deposit.

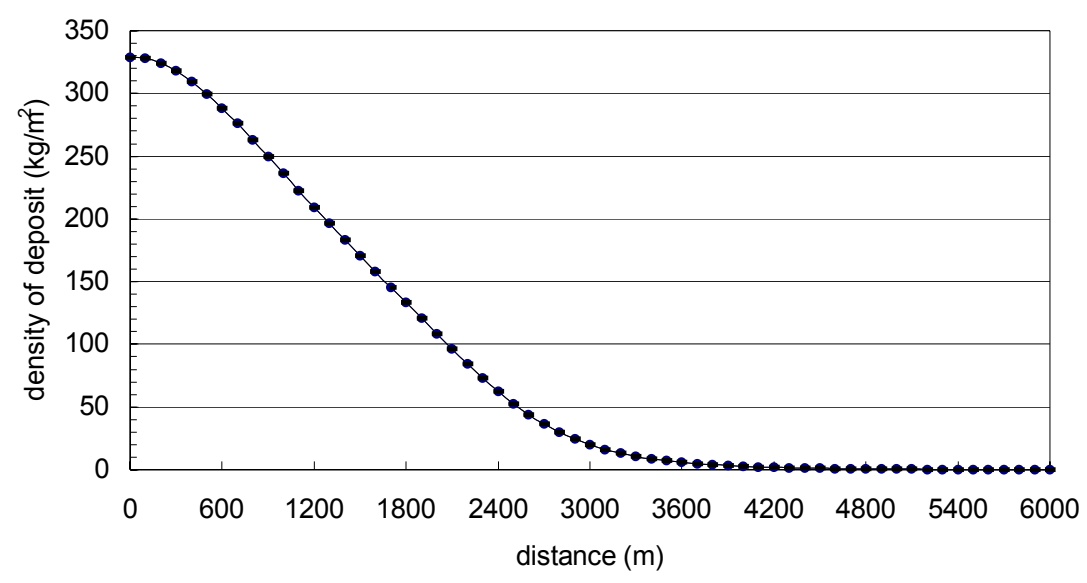

Figure 3 . The density of the target deposit along the $x$ direction The initial volume per unit width of the current is $10^{4} \mathrm{~m}^{2}$ and initial volume fraction of particle is 0.02 . 


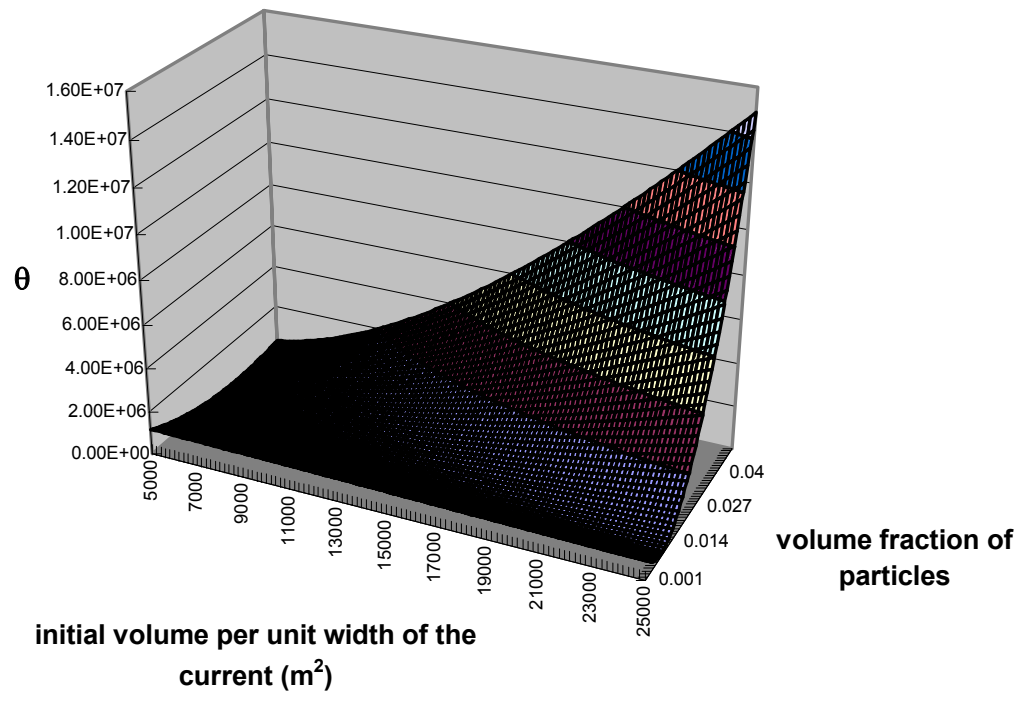

Figure 4. Optimization objective surface

\section{RESULTS AND DISCUSSION - PRELIMINARY SENSITIVITY STUDY OF PARAMETER ESTIMATION FROM TURBIDITE DATA}

\section{Effect of number of point observations}

In reality, especially during the initial development of a field, little information is available on the density of deposit. So we decrease $M$ to see this effect. Decreasing $M$ to 3 influences the result. We were unable to find any starting point that returned the target $q$ and $\phi$ for $M=3$. For $M>3$, given proper iteration starting values, the target $q$ and $\phi$ were returned.

Occasionally Solver will not converge for a given starting point. For example, wee find that if $q_{0}=100 \mathrm{~m}^{2}$ and $\phi_{0}=0.001$, there is no convergence when $M=3$ but there is when $M=60$. There is a physical reason for this behavior; the starting values are so far from the target values that $w=0$ at some of the locations. These values inhibit convergence and indicate that the starting points must be chosen to be fairly close to the final values. That is, like all non-linear optimization problems, a good initial guess is needed.

\section{Influence of measurement error}

In the field, measured values may be inaccurate. To investigate this effect we added noise to the $w(x)$ target. For many of the cases, the optimized $\hat{q}$ and $\hat{\phi}$ proved to be sensitive to the starting values, $q_{0}$ and $\phi_{0}$, used in Solver. The relative error of $\hat{q}$ and $\hat{\phi}$ can be expressed as 


$$
\begin{aligned}
& \text { relativeerrorof } \hat{q}=\frac{|\hat{q}-q|}{q} \times 100 \% \\
& \text { relativeerror of } \hat{\phi}=\frac{|\hat{\phi}-\phi|}{\phi} \times 100 \%
\end{aligned}
$$

In the following tables the relative error of the converged $\hat{q}$ and $\hat{\phi}$ are the arithmetic average of three realizations with different $q_{0}$ and $\phi_{0}$.

\section{Perturbing the density measurements}

First, we added drift to the original target density as $\alpha \cdot(\operatorname{rand}()-0.5) . \quad \alpha$ is a coefficient $(\geq 1)$ equal to $1,10,20 \ldots$ Rand is the Excel function that generates a uniformly distributed random number on the interval $(0,1)$. We hope to find the maximum $\alpha$ for which Solver will continue to return reasonably accurate target values $\hat{q}$ and $\hat{\phi}$. If the perturbed target density becomes negative after adding noise, we set it to zero. Adding noise will influence the returned value (See Table 2). For $M=60$ data points $\alpha$ will reach 70 before the optimization returned values that are more than $10 \%$ in error. The results are shown in Figure 5 and Table 2.

The perturbation here is additive and has a zero mean regardless of the value of $\alpha$. This means that the total volume of the target deposition is unaffected by the perturbation until the effects of the truncation become large. At some point, evidently around $\alpha=70$, the perturbed target has lost so much mass to truncation that it is impossible for the optimization to return the target $q$ and $\phi$, though it continues to converge.

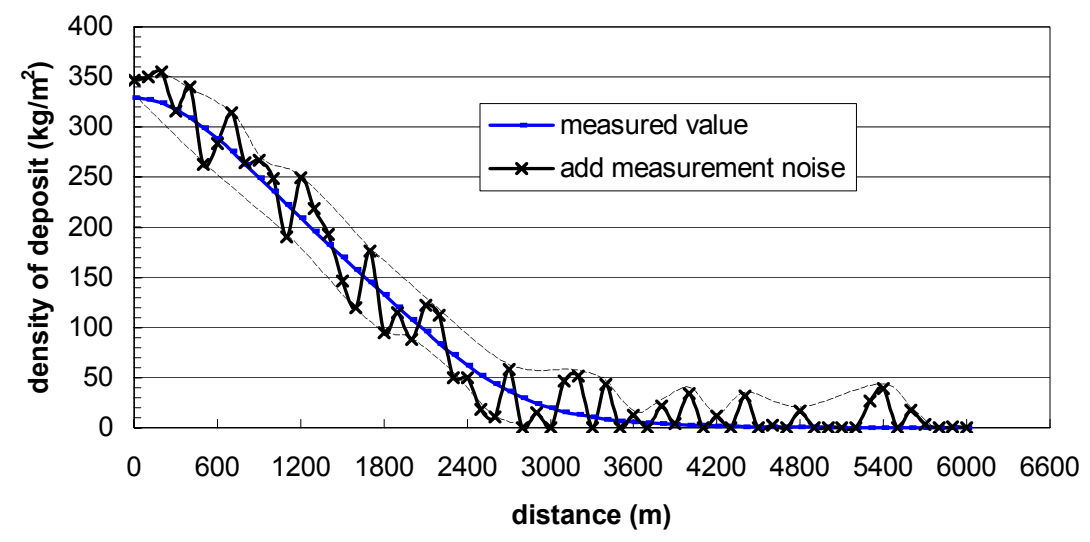

Figure 5. Plot of $w(x)+\alpha \cdot(\operatorname{rand}()-0.5)$ one random series of $\alpha=80$

The range of noise is from -40 to +40 . 
Table 2 the result of adding noise as $\alpha \cdot(\operatorname{rand}()-0.5)(M=60)$

\begin{tabular}{|c|c|c|c|c|c|c|c|c|c|}
\hline$\alpha$ & 1 & 10 & 20 & 30 & 40 & 50 & 60 & 70 & 80 \\
\hline $\begin{array}{c}\text { Average relative } \\
\text { error of } \hat{q}(\%)\end{array}$ & 0.04 & 0.56 & 1.33 & 3.47 & 5.55 & 2.72 & 5.89 & 6.94 & 10.78 \\
\hline $\begin{array}{c}\text { Average relative } \\
\text { error of } \hat{\phi}(\%)\end{array}$ & 0.05 & 0.71 & 0.88 & 2.27 & 5.10 & 2.43 & 4.05 & 4.01 & 8.98 \\
\hline
\end{tabular}

Another type of perturbation is multiplicative. Now we add noise proportional to the target as $\lambda \cdot w(x) \cdot(\operatorname{rand}()-0.5) . w(x)$ is the original target density of the deposit at location $x . \lambda$ is a coefficient $(\geq 0)$ equal to $5 \%, 10 \%, 15 \% \ldots$ each time. Now there will no negative densities and the noise distribution along the distance looks like a cone. For $M=60$ data points $\lambda$ will reach $35 \%$ before the optimization results deviate from the target values by more than $5 \%$.

Comparing the absolute noise for points nearest the source (along the $x$ direction) the multiplicative perturbations are larger than for the additive perturbations, which means that larger deposit densities can tolerate larger noise. The graph and results are also shown in Figure 6 and Table 3.

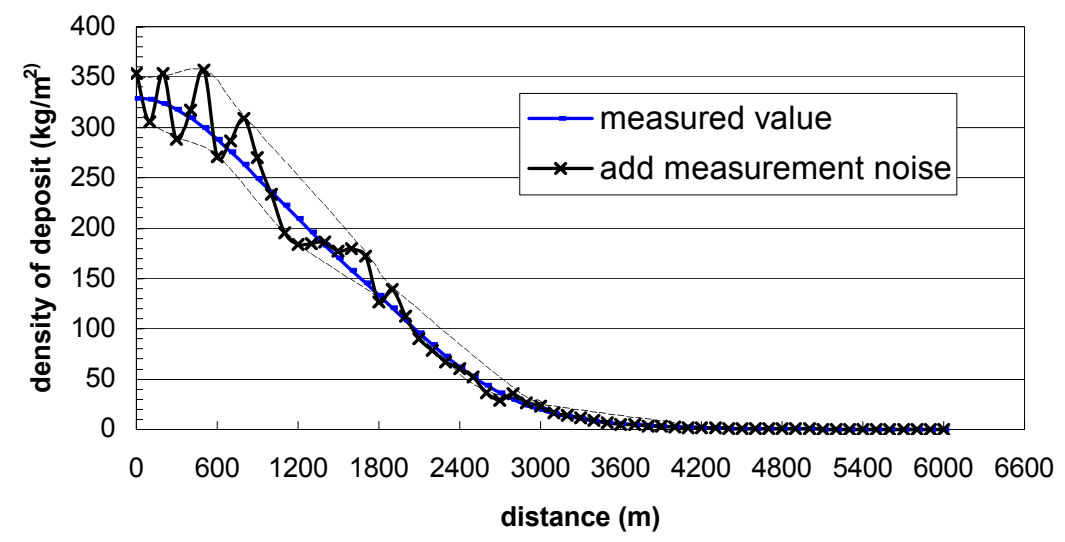

Figure 6. Plot of $w(x)+\lambda \cdot w(x) \cdot(\operatorname{rand}()-0.5)$ one random series of $\lambda=40 \%$ The range of noise is from -0.2 times measurement to +0.2 times measurement. 
Table 3. The results of adding noise as $\lambda \cdot w(x) \cdot(\operatorname{rand}()-0.5)(M=60)$

\begin{tabular}{|c|c|c|c|c|c|c|c|}
\hline$\lambda$ & $5 \%$ & $10 \%$ & $15 \%$ & $20 \%$ & $25 \%$ & $30 \%$ & $35 \%$ \\
\hline $\begin{array}{c}\text { Average } \\
\text { relative } \\
\text { error of } \hat{q}(\%)\end{array}$ & 0.66 & 1.52 & 1.29 & 2.23 & 4.39 & 3.79 & 4.32 \\
\hline $\begin{array}{c}\text { Average } \\
\text { relative } \\
\text { error of } \hat{\phi} \\
(\%)\end{array}$ & 0.76 & 2.14 & 1.97 & 3.52 & 4.67 & 5.29 & 6.09 \\
\hline
\end{tabular}

Next, we look at reducing $M$ on the perturbed targets. The results are in Table 4 and Table 5. Reducing $M$ leads to more error because the values of and . both decrease at the same relative errors. Thus, and as is to be expected, the results are more sensitive to errors when there are few measurement points.

Table 4. The results of adding noise as $\alpha \cdot(\operatorname{rand}()-0.5)(M=3)$

\begin{tabular}{|c|c|c|c|c|c|}
\hline$\alpha$ & 1 & 10 & 20 & 30 & 40 \\
\hline $\begin{array}{c}\text { Average relative error } \\
\text { of } \hat{q}(\%)\end{array}$ & 0.36 & 2.04 & 4.68 & 6.96 & 18.48 \\
\hline $\begin{array}{c}\text { Average relative error } \\
\text { of } \hat{\phi}(\%)\end{array}$ & 0.19 & 1.29 & 6.42 & 12.31 & 9.67 \\
\hline
\end{tabular}

Table 5. The results of adding noise as $\lambda \cdot w(x) \cdot(\operatorname{rand}()-0.5)(M=3)$

\begin{tabular}{|c|c|c|c|c|c|}
\hline$\lambda$ & $1 \%$ & $5 \%$ & $15 \%$ & $20 \%$ & $25 \%$ \\
\hline $\begin{array}{c}\text { average relative error } \\
\text { of } \hat{q}(\%)\end{array}$ & 0.43 & 2.31 & 3.09 & 8.66 & 9.54 \\
\hline $\begin{array}{c}\text { average relative error } \\
\text { of } \hat{\phi}(\%)\end{array}$ & 0.79 & 3.75 & 6.14 & 14.61 & 15.10 \\
\hline
\end{tabular}

\section{Perturbing the locations of measurements}

Locations of the measurement points may be exact. So drift is not avoidable. However from inspection of Eq. (22) it is not difficult to see that the influence of error in $x$ on the density of deposit is not large. Perturbing the locations should have a small effect on returned values both for $M=60$ and $M=3$. (Recall that all point measurements are equally spaced.) This indicates that the major source of a problematic error will be in 
the thickness measurements, not the locations. The results of calculation are shown in Table 6 and Table 7 to demonstrate this conclusion.

Table 6 . The result of adding noise as $\alpha \cdot(\operatorname{rand}()-0.5)$ on locations $(M=60)$

\begin{tabular}{|c|c|c|c|c|c|}
\hline$\alpha$ & 10 & 20 & 40 & 60 & 80 \\
\hline $\begin{array}{c}\text { average relative error } \\
\text { of } \hat{q}(\%)\end{array}$ & 0.06 & 0.13 & 0.31 & 0.38 & 0.62 \\
\hline $\begin{array}{c}\text { Average relative error } \\
\text { of } \hat{\phi}(\%)\end{array}$ & 0.06 & 0.10 & 0.17 & 0.37 & 0.51 \\
\hline
\end{tabular}

Table 7. The result of adding noise as $\alpha \cdot(\operatorname{rand}()-0.5)$ on locations $(M=3)$

\begin{tabular}{|c|c|c|c|c|c|}
\hline$\alpha$ & 10 & 20 & 40 & 60 & 80 \\
\hline $\begin{array}{c}\text { Average relative error } \\
\text { of } \hat{q}(\%)\end{array}$ & 0.42 & 0.72 & 1.76 & 1.19 & 2.62 \\
\hline $\begin{array}{c}\text { Average relative error } \\
\text { of } \hat{\phi}(\%)\end{array}$ & 0.47 & 0.65 & 1.98 & 0.69 & 2.75 \\
\hline
\end{tabular}




\section{CONCLUSIONS}

In this report we discuss work on two aspects of this project: the creation of a simulation of the turbidity flow and deposit and the inference of the initial conditions of such a flow based on a few measurements from cores from the deposit.

Regarding the first part of the work, we have developed and tested a new FEMbased algorithm to simulate the flow of two-dimensional concentrated viscous turbidity currents over a horizontal non-erodible bed. We have found that

- This new FEM algorithm is vastly superior to our previous approach. It accommodates variable grid distribution, which makes simulations over large domains with high degree of accuracy in regions of interest possible.

- The use of weak variational formulation and the symmetric form of matrices resulting from the implementation reduces the computation time.

- Lastly, the accuracy of particle concentration profile obtained from FEMbased flux-corrected transport algorithm is better than that obtained from the finite-volume based algorithm.

In short we have established a method most suitable to this problem.

From the preliminary study of parameter estimation of the initial conditions based on measurements of a synthetic deposit, we have found that

- We have demonstrated that an inverse method is feasible by performing optimizations using Solver. We must use the scaling option in Solver for convergence, and we speculate such a procedure will be necessary in the final application.

- When adding both additive and multiplicative perturbations to the density, the average relative error in the returned values will increase with the size of the perturbation.

- Adding noise to the locations plays a small role to the results.

- To perform the optimization we must choose fairly accurate starting values when the number of measurement points is small.

\section{FUTURE WORK}

For the next six months, we aim to complete the following tasks:

- Complete testing on 2-D simulation for high Reynolds number flows

- Perform parameter study for 2D simulations over horizontal non-erodible bed for various $N_{B}$ and $\varphi_{\text {in }}$ values.

- Perform parameter study for 2D simulations over an inclined surface.

- Perform parameter study for 2D simulations over an erodible bed.

- Begin conversion of 2D simulation to 3D.

- Adopt method inverse method to turbidite data created by $2 \mathrm{D}$ numerical simulation rather than the algebraic form used here.

- Include polydispersity of particle sizes. 


\section{REFERENCES}

Bonnecaze, R.T., Huppert, H.E.\& Lister, J.R.1996 Patterns of sedimentation from polydispersed turbidity currents. Proc. R. Soc. Lond. 452, 2247-2261.

Codina, R. \& Blasco, J. (1997), "A finite element formulation for the stokes problem allowing equal velocity-pressure interpolation", Comput. Methods Appl. Mech. Engrg., 143, 373 - 391.

Davis, R. H. \& Acrivos, A. (1985), "Sedimentation of noncolloidal particles at low Reynolds numbers", Annu. Rev. Fluid Mech., 17, $91-118$.

Georghiou, G. E., Morrow, R. \& Metaxos, A. C. (1999), "An improved finite-element flux-corrected transport algorithm”, J. Comput. Phys., 148, 605-620.

Georghiou, G. E., Morrow, R. \& Metaxos, A. C. (2000), "A two-dimensional finiteelement flux-corrected transport algorithm for the solution of gas discharge prolems", J. Phys. D: Appl. Phys., 33, 2453 - 2466.

Morris, J. F. \& Boulay, F. (1999), "Curvilinear flows of noncolloidal suspensions: The role of normal stresses", J. Rheol., 43, 1213 - 1237.

Morris, J. F. \& Brady, J. F. (1998), "Pressure-driven flow of a suspension: Buoyancy effects", Int. J. Multiphase Flow, 24(1), 105 - 130.

Nonino, C. \& Comini, G. (1997), "An equal-order velocity-pressure algorithm for incompressible thermal flows, Part 1: Formulation", Numer. Heat Transfer, Part B, 32, $1-15$.

Nott, P. R. and Brady, J. F. (1994), "Pressure-driven flow of suspensions: simulation and theory", J Fluid Mech., 275, 157 - 199.

Williams, P. T. \& Baker, A. J. (1966), "Incompressible computational fluid dynamics and continuity constraint method for the three-dimensional Navier-Stokes equations", Numer. Heat Transfer, Part B, 29, 137 - 273. 